\title{
Revista Colombiana de

\section{Perfil lipídico y consumo de frutas y verduras en un grupo de jóvenes de 10 a 19 años, según el índice de masa corporal}

\author{
Beatriz E. Parra ${ }^{a, *}$, Luz M. Manjarrés a , Claudia M. Velásqueza \\ Gloria M. Agudelo ${ }^{b}$, Alejandro Estrada ${ }^{a}$, Rosa M. Uscátegui ${ }^{a}$, Fredy A. Patiño ${ }^{c}$, \\ Gabriel de J. Bedoya ${ }^{d}$ y María V. Parra ${ }^{d}$

\footnotetext{
a Escuela de Nutrición y Dietética, Universidad de Antioquia, Medellín, Colombia

b Grupo Vidarium, Centro de Investigación y Nutrición, Salud y Bienestar del Grupo Nutresa, Medellín, Colombia

c Instituto de Educación Física, Universidad de Antioquia, Medellín, Colombia
} \\ ' Genética Molecular GENMOL, Universidad de Antioquia, Medellín, Colombia
}

Recibido el 23 de octubre de 2013; aceptado el 17 de octubre de 2014

Disponible en Internet el 3 de marzo de 2015

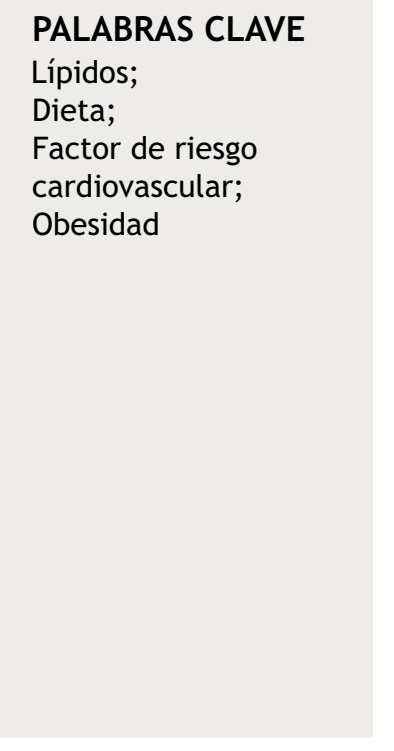

\begin{abstract}
Resumen
Introducción: El exceso de peso en niños y adolescentes es una pandemia que aumenta el riesgo de mortalidad por enfermedades crónicas no transmisibles. Se ha estimado que aun en etapas tempranas de la vida, el exceso de peso se asocia con alteraciones metabólicas; sin embargo, es necesario establecer si en nuestro medio estas alteraciones se evidencian en población menor de 19 años.

Objetivo: Comparar el perfil lipídico y la ingesta de frutas y verduras, según el índice de masa corporal, en un grupo de jóvenes de 10 a 19 años, de la empresa promotora de salud SURA, sede de Medellín.

Métodos: Estudio de corte transversal, en el que participaron 103 jóvenes obesos, 120 con sobrepeso y 214 con un índice de masa corporal normal. En condiciones basales y ayuno de 10 horas, se evaluó colesterol total, c-LDL, c-HDL y triglicéridos. La ingesta de verduras y frutas se determinó mediante recordatorio de 24 horas.

Resultados: Los datos de c-LDL en el grupo de obesos, sobrepeso y control fueron, respectivamente: $95 \pm 32,96 \pm 53$ y $80 \pm 24 \mathrm{mg} / \mathrm{dL}(\mathrm{p}=0,000)$; para los mismos grupos, los datos de triglicéridos fueron: $116 \pm 65,112 \pm 69$ y $88 \pm 52 \mathrm{mg} / \mathrm{dL}(p=0,000)$. El c-HDL en los 3 grupos fue: $52 \pm 14,53 \pm 12$ y $56 \pm 14 \mathrm{mg} / \mathrm{dL}(\mathrm{p}=0,013)$, respectivamente. Se evidenció una asociación significativa entre el mayor consumo de frutas y la menor concentración de triglicéridos entre los participantes con sobrepeso $(p=0,035)$. No fue habitual la ingesta de frutas y verduras.
\end{abstract}

\footnotetext{
* Autor para correspondencia.

Correo electrónico: beatriz.parra@udea.edu.co (B.E. Parra).
} 
Conclusiones: El alto índice de masa corporal promueve un perfil lipídico aterogénico. Son necesarias acciones para promover hábitos alimentarios saludables.

(C) 2015 Sociedad Colombiana de Cardiología y Cirugía Cardiovascular. Publicado por Elsevier España, S.L.U. Este es un artículo Open Access bajo la licencia CC BY-NC-ND (http://creativecommons.org/licenses/by-nc-nd/4.0/).

\section{KEYWORDS}

Lipids;

Diet;

Cardiovascular risk

factor;

Obesity
Lipid profile and consumption of fruits and vegetables in a youth group from 10-19 years according to body mass index

\begin{abstract}
Introduction: Childhood overweight is a pandemic that increases the risk of chronic noncommunicable diseases. It has been estimated that being overweight is associated with metabolic disorders; even in early stages of life. However, it is necessary to establish whether this association is observed in Colombian population between 10-19 years old.

Objective: To compare lipid profile and fruit and vegetable intakes, according to body mass index, in subjects aged 10-19 in a health insurance company (SURA) from Medellin.

Methods: One hundred an three obese, 120 overweight, and 214 children with normal body mass index participated in a cross-sectional study. We compared total cholesterol, LDL-C, HDL-C and triglycerides between groups; at baseline and after 10-hour fasting period. Fruit and vegetable intakes were determined by 24-hour dietary record.

Results: LDL-C levels in obese, overweight and control group were, respectively: $95 \pm 32,96 \pm$ 53 and $80 \pm 24 \mathrm{mg} / \mathrm{dL}(\mathrm{P}=.000)$. Triglycerides levels in the same groups were: $116 \pm 65,112 \pm$ 69 and $88 \pm 52 \mathrm{mg} / \mathrm{dL}(P=.000)$. HDL-C levels in the 3 groups were: $52 \pm 14,53 \pm 12$ and $56 \pm 14$ $\mathrm{mg} / \mathrm{dL}(\mathrm{P}=.013)$. A significant inverse association between fruit intake and triglyceride levels in overweight participants was observed $(P=.035)$. Fruit and vegetable intake were unusual. Conclusions: A high body mass index promotes an atherogenic lipid profile. It is necessary to implement actions to promote healthy habits associated with diet.

(c) 2015 Sociedad Colombiana de Cardiología y Cirugía Cardiovascular. Published by Elsevier España, S.L.U. This is an open access article under the CC BY-NC-ND license (http://creativecommons.org/licenses/by-nc-nd/4.0/).
\end{abstract}

\section{Introducción}

El exceso de peso es una epidemia que afecta a la población tanto de países desarrollados como en vía de desarrollo ${ }^{1}$, $y$ es un factor determinante de la morbimortalidad por enfermedades crónicas no transmisibles. América del Sur se encuentra en una etapa de transición epidemiológica, en la que se observa un cambio en la predominancia de la mortalidad, desde aquella ocasionada por enfermedades infecciosas agudas, hasta la producida por enfermedades crónicas $^{2}$. El informe de los indicadores básicos de la situación de salud en las Américas 2012 muestra una tasa de mortalidad por enfermedades infecciosas del 55,4 por 100.000 habitantes, y para enfermedad isquémica y cerebrovascular, del 57,3 y 42,8 por 100.000 habitantes, respectivamente ${ }^{3}$.

Resultados de estudios epidemiológicos y experimentales han asociado el origen temprano del desarrollo de enfermedades crónicas, especialmente cardiovasculares, con el comienzo precoz del proceso aterosclerótico en la infancia y su progreso acelerado durante la vida. Aun cuando se reconoce que el componente genético aumenta la susceptibilidad del individuo al desarrollo de estas enfermedades, está claro que los factores medioambientales, como la dieta y la actividad física, son fundamentales para determinar su evolución y desenlace ${ }^{4}$.
Dentro de los principales factores de riesgo identificados por la Organización Mundial de la Salud como claves para el desarrollo de enfermedades crónicas, 5 están estrechamente relacionados con la alimentación y el ejercicio físico: la obesidad, el sedentarismo, la hipertensión arterial, la hipercolesterolemia y el consumo insuficiente de frutas y verduras ${ }^{5}$.

Se ha considerado que el consumo de frutas y verduras reduce la obesidad debido a que aumenta la cantidad de fibra, disminuye la ingesta de grasa y la densidad energética de la dieta, e incrementa la sensación de saciedad ${ }^{1,6}$; al respecto, Lock et al. hallaron que más de 2,5 millones de muertes en el mundo y el $31 \%$ de las enfermedades cardiovasculares se pueden atribuir a un consumo inadecuado de frutas y verduras, factor determinante tanto en países desarrollados como en vías de desarrollo ${ }^{7}$.

Diferentes estudios en jóvenes muestran una tendencia a estilos de vida caracterizados por hábitos alimentarios inadecuados y sedentarismo, al tiempo que otras investigaciones evidencian un aumento en la prevalencia de exceso de peso y alteraciones metabólicas a más temprana edad. El diagnóstico de sobrepeso u obesidad por sí solo compromete la calidad de vida durante la niñez y la adolescencia e incrementa el riesgo de morbilidad psicosocial, así como de complicaciones cardiovasculares y de diabetes mellitus. En Colombia, este problema está aumentando, lo cual se confirma al comparar los resultados de la Encuesta Nacional de 
la Situación Nutricional 2005 con la realizada en 2010; en la primera, se reportó que un $10,3 \%$ de los jóvenes entre 10 y 17 años presentó exceso de peso, mientras que en la Encuesta Nacional de la Situación Nutricional 2010, la prevalencia fue de un $16,7 \%$ en el mismo grupo etario ${ }^{8,9}$. Específicamente, en Medellín, un estudio realizado en 2002 encontró una prevalencia de sobrepeso del $9,3 \%$, y de obesidad, del $4,6 \%{ }^{10}$. Posteriormente, en 2009 , el perfil alimentario y nutricional en esta misma ciudad reportó en la población de 11 a 18 años una prevalencia de sobrepeso y de obesidad del 15,2 y $5,6 \%$, respectivamente ${ }^{11}$.

Como único problema de salud, el exceso de peso se encuentra entre los factores de riesgo más importantes para enfermedades crónicas, de las cuales la diabetes mellitus y las enfermedades del corazón serán responsables de la mortalidad de 388 millones de personas en el mundo entero en la próxima década, si no se establecen acciones para su prevención $^{12}$.

Por lo anterior, este estudio tuvo como objetivo comparar el perfil lipídico y la ingesta de frutas y verduras según el índice de masa corporal (IMC), en un grupo de jóvenes de 10 a 19 años de la empresa promotora de salud SURA, de Medellín (Colombia).

\section{Material y métodos}

Estudio de corte transversal derivado del macroproyecto «Síndrome metabólico en jóvenes con exceso de peso: identificación de factores de riesgo y evaluación de una intervención», para el cual se calculó una muestra probabilística de 1.060 jóvenes de 10 a 19 años de una empresa promotora de salud, seleccionados por muestreo aleatorio para identificar la prevalencia de exceso de peso ${ }^{13}$. En el presente estudio la muestra estuvo constituida por 437 jóvenes de los evaluados anteriormente, clasificados en 3 grupos: obesos $(n=103)$, sobrepeso $(n=120)$ y grupo control (peso adecuado, $n=214$ ), emparejados por edad, maduración puberal, escolaridad y estrato socioeconómico, a quienes les fue evaluado el perfil lipídico y la ingesta de verduras y frutas.

Se excluyeron jóvenes que estuvieran en tratamiento médico o nutricional para el manejo de dislipidemia, que consumieran corticosteroides y tomaran hormonas tiroideas, o aquellos con diagnóstico de diabetes, enfermedades genéticas, incapacidad física permanente o temporal, en gestación o lactancia y deportistas de alto rendimiento.

Una vez seleccionada la muestra, aceptada la participación en el estudio por el joven y obtenida la autorización de padres o acudiente, se inició el proceso de recolección de la siguiente información.

\section{Encuesta de consumo de alimentos}

La ingesta alimentaria se evaluó en el domicilio del joven mediante un recordatorio de $24 \mathrm{~h}$ distribuido de forma aleatoria durante los diferentes días de la semana. Se usaron modelos de alimentos, figuras geométricas y álbum de fotografías en tamaño real para mayor exactitud ${ }^{14}$. El recordatorio de $24 \mathrm{~h}$ fue realizado por estudiantes de nutrición entrenados y estandarizados previamente en el proceso; además, a cada joven se le preguntó si en el último mes consumió frutas y verduras diariamente.

\section{Pruebas bioquímicas}

En condiciones basales y ayuno de $10 \mathrm{~h}$, se obtuvo una muestra de $3 \mathrm{~mL}$ de sangre venosa para determinar el perfil lipídico con la evaluación de colesterol total (CT), c-LDL, c-HDL y triglicéridos (TG) por espectrofotometría en fotocolorímetro RA50 (Bayer, serie 71663); se utilizaron kits colorimétricos enzimáticos específicos (BioSystems Reagents and Instruments). Para el CT y los TG se utilizó el método oxidasa/peroxidasa; para el c-HDL y el c-LDL se aplicó el método directo con detergente, que igualmente emplea las reacciones oxidasa/peroxidasa.

De acuerdo con el Panel de Expertos en Colesterol Sanguíneo del Programa Nacional de Educación en Colesterol, para los niños mayores de 2 años y adolescentes, las concentraciones de CT y de c-LDL consideradas como altas son $\geq 200$ $\mathrm{y} \geq 130 \mathrm{mg} / \mathrm{dL}$, respectivamente, mientras que se consideró $\mathrm{c}-\mathrm{HDL}<40 \mathrm{mg} / \mathrm{dL}$ como un valor bajo y TG $>110 \mathrm{mg} / \mathrm{dL}$ como alto $^{15}$.

Con el índice de Castelli (CT/c-HDL) se determinó el factor de riesgo cardiovascular y la clasificación fue positiva (presencia del riesgo) cuando el resultado de este cociente fue mayor de 5 para hombres y mayor de 4,5 en mujeres. Adicionalmente, se estimó el índice aterogénico (c-LDL/c$\mathrm{HDL}$ ), con un valor de riesgo para hombres mayor de $3,5 \mathrm{y}$ para mujeres superior a $3,0^{16}$.

\section{Manejo ético de la investigación}

La investigación se clasificó en riesgo mínimo según el Ministerio de Salud de Colombia en la Resolución número 008430, artículo 11, de octubre de 1993, y fue aprobada por el Comité de Bioética de la Sede de Investigación Universitaria de la Universidad de Antioquia (Medellín, Colombia). Los participantes, padres o acudientes, firmaron el consentimiento informado, el cual cumplió con los principios éticos de la Declaración de Helsinki para investigaciones médicas en seres humanos ${ }^{17}$.

\section{Plan de análisis}

Los datos de consumo de alimentos se ingresaron en el Programa de Evaluación de la Ingesta Dietética EVINDI $\mathrm{v} 4^{18}$, el cual permitió obtener información de la cantidad de alimentos ingeridos de los diferentes grupos. El análisis estadístico se realizó con el programa Statistical Package for the Social Sciences ${ }^{\circledR}$ versión 18.0. Se comprobó la normalidad de las variables continuas mediante la prueba de Kolmogorov-Smirnov y las asociaciones se evaluaron mediante las pruebas de $\mathrm{Chi}^{2}$ y Kruskal-Wallis; para esta última, las comparaciones múltiples entre categorías de IMC se efectuaron mediante la prueba $U$ de Mann-Whitney.

\section{Resultados}

La muestra estuvo conformada por 437 jóvenes con una edad media de $13 \pm 2,2$ años; el $68,9 \%$ tenía entre 10 y 13,9 años y 
Tabla 1 Características generales y maduración sexual en los jóvenes de la empresa promotora de salud SURA de acuerdo con el índice de masa corporal. Medellín, Colombia

\begin{tabular}{llll}
\hline & Adecuado $(\mathrm{n}=214)$ & Sobrepeso $(\mathrm{n}=120)$ & Obesidad $(\mathrm{n}=103)$ \\
\hline $\begin{array}{l}\text { Edad en años, media } \pm D E \\
\text { Estatura en } \mathrm{cm}, \text { media } \pm D E\end{array}$ & $13,4 \pm 2,2$ & $13,3 \pm 2,1$ & $13,3 \pm 2,2$ \\
$\begin{array}{l}\text { Sexo, } n \text { (\%) } \\
\quad 152,7 \pm 12,3\end{array}$ & $155,5 \pm 10,9$ & $155,0 \pm 12,2$ \\
$\quad \begin{array}{l}\text { Masculino } \\
\text { Femenino }\end{array}$ & $111(51,9)$ & $53(44,2)$ & $63(61,2)$ \\
Maduración puberal, $n(\%)$ & $103(48,1)$ & $67(55,8)$ & $40(38,8)$ \\
$\quad$ & & & \\
$\quad \begin{array}{l}\text { Prepúber } \\
\text { Púber }\end{array}$ & $54(25,2)$ & $19(15,8)$ & $21(20,4)$ \\
Pospúber & $72(33,6)$ & $40(33,3)$ & $41(39,8)$ \\
\hline
\end{tabular}

a Según la prueba de Kruskal-Wallis.

b Según la prueba de $\mathrm{Chi}^{2}$.

el $51,9 \%$ era de sexo masculino. La tabla 1 resume las características generales y de maduración sexual de los jóvenes de acuerdo con el IMC, y muestra que los grupos fueron homogéneos por edad y maduración puberal; en el caso del sexo, en el que se hallaron diferencias significativas según el IMC, se probó la independencia en cada una de las variables analizadas.

La tabla 2 muestra la distribución de los lípidos sanguíneos según la clasificación del IMC. El CT fue el único indicador que no evidenció diferencias significativas según el IMC; el C-LDL y los TG fueron significativamente mayores en los jóvenes con obesidad y sobrepeso en comparación con los de peso adecuado, mientras que el c-HDL fue menor. Los resultados fueron independientes del sexo para el CT y el c-LDL, pero los niños no presentaron diferencias estadísticamente significativas en el $\mathrm{C}$-HDL de acuerdo con el IMC $(p=0,402)$, ni en los TG entre los que se clasificaron con sobrepeso y peso adecuado $(p=0,062)$ (tabla 2$)$.

Independientemente del IMC, la mitad de los jóvenes se clasificó con hipercolesterolemia, mientras que los otros indicadores del perfil lipídico estuvieron más alterados en los jóvenes con exceso de peso (tabla 3).

Al estimar el índice de Castelli se encontró riesgo en el $7,9 \%$ de los niños y en el $9,8 \%$ de las niñas $(p=0,448)$. De acuerdo con la clasificación por IMC, se halló que el $55,5 \%$ de los niños y el 76,2\% de las niñas con alteración del índice de Castelli presentaron sobrepeso $(p=0,173)$. Al calcular el índice aterogénico se encontró que el $6,5 \%$ de las niñas y el $1,3 \%$ de los niños presentaron riesgo $(p=0,004)$, de los cuales el $85,7 \%$ de las niñas y el $100 \%$ de los niños tenían exceso de peso (sobrepeso u obesidad).

La baja ingesta de frutas y verduras en los adolescentes fue habitual, pues al preguntar sobre la frecuencia diaria de su consumo en el último mes, el $66,6 \%$ reportó que no ingirió frutas y el $73,5 \%$ que no consumió verduras. La tabla 4 muestra el bajo porcentaje de jóvenes que ingirió alguna fruta y/o verdura el día anterior, la poca cantidad consumida y la falta de variedad.

Cuando se comparó la distribución de los lípidos séricos según el consumo de frutas y verduras por terciles, dentro de cada categoría de IMC, la única diferencia estadísticamente significativa se encontró en la concentración de TG de los jóvenes con sobrepeso, en quienes el menor valor se observó en aquellos que se ubicaron en el tertil superior de consumo de frutas (tabla 5).

\section{Discusión}

Las enfermedades isquémicas del corazón y las cerebrovasculares constituyen las primeras causas de muerte en Colombia ${ }^{19}$, y dentro de los factores de riesgo modificables

Tabla 2 Distribución de lípidos séricos según el índice de masa corporal en los jóvenes de 10 a 19 años de la empresa promotora de salud SURA. Medellín, Colombia

\begin{tabular}{|c|c|c|c|c|c|c|c|}
\hline & \multicolumn{2}{|c|}{ Adecuado $(n=124)$} & \multicolumn{2}{|c|}{ Sobrepeso $(n=120)$} & \multicolumn{2}{|c|}{ Obesidad $(n=130)$} & \\
\hline & Media $\pm \mathrm{DE}$ & M & Media $\pm \mathrm{DE}$ & M & Media $\pm \mathrm{DE}$ & M & \\
\hline Colesterol total ${ }^{\mathrm{a}}, \mathrm{mg} / \mathrm{dL}$ & $174 \pm 40$ & 173 & $181 \pm 45$ & 175 & $133 \pm 2,2$ & 174 & 0,551 \\
\hline Triglicéridos, mg/dL & $88 \pm 52$ & 78 & $112 \pm 69$ & $97^{\mathrm{b}}$ & $116 \pm 65$ & $101^{\mathrm{b}}$ & 0,000 \\
\hline $\mathrm{c}-\mathrm{HDL}, \mathrm{mg} / \mathrm{dL}$ & $56 \pm 14$ & 55 & $53 \pm 12$ & 52 & $52 \pm 14$ & $50^{\mathrm{b}}$ & 0,013 \\
\hline $\mathrm{c}-\mathrm{LDL}, \mathrm{mg} / \mathrm{dL}$ & $80 \pm 24$ & 75 & $96 \pm 35$ & $93^{b}$ & $95 \pm 32$ & $90^{\mathrm{b}}$ & 0,000 \\
\hline
\end{tabular}

c-HDL: colesterol ligado a lipoproteínas de alta densidad; c-LDL: colesterol ligado a lipoproteínas de baja densidad; DE: desviación estándar; M: mediana.

a Según la prueba de Kruskal-Wallis.

b Presentaron diferencias estadísticas con relación al grupo con índice de masa corporal adecuado según la prueba de Kruskal-Wallis $(p<0,05)$. 
Tabla 3 Clasificación del perfil lipídico según el índice de masa corporal de los jóvenes de 10 a 19 años de la empresa promotora de salud SURA. Medellín, Colombia

\begin{tabular}{|c|c|c|c|c|c|c|c|c|}
\hline \multirow[b]{3}{*}{ Lípidos séricos } & \multirow[b]{3}{*}{ Clasificación } & \multicolumn{6}{|c|}{ Distribución según IMC } & \multirow[t]{3}{*}{$\mathrm{p}^{\mathrm{a}}$} \\
\hline & & \multicolumn{2}{|c|}{$\begin{array}{l}\text { Adecuado } \\
(n=214)\end{array}$} & \multicolumn{2}{|c|}{$\begin{array}{l}\text { Sobrepeso } \\
(n=120)\end{array}$} & \multicolumn{2}{|c|}{$\begin{array}{l}\text { Obesidad } \\
(n=103)\end{array}$} & \\
\hline & & $\mathrm{n}$ & $\%$ & $\mathrm{n}$ & $\%$ & $\mathrm{n}$ & $\%$ & \\
\hline \multirow[t]{2}{*}{ Colesterol total } & Normal & 101 & 47,2 & 58 & 48,3 & 47 & 45,6 & 0,922 \\
\hline & Alto & 113 & 52,8 & 62 & 51,7 & 56 & 54,4 & \\
\hline \multirow[t]{2}{*}{ Triglicéridos } & Normal & 173 & 80,8 & 71 & 59,2 & 60 & 58,3 & 0,000 \\
\hline & Alto & 41 & 19,2 & 49 & 40,8 & 43 & 41,7 & \\
\hline \multirow[t]{2}{*}{$\mathrm{c}-\mathrm{HDL}$} & Normal & 190 & 88,8 & 106 & 88,3 & 82 & 79,6 & 0,064 \\
\hline & Alto & 24 & 11,2 & 14 & 11,7 & 21 & 20,4 & \\
\hline \multirow[t]{2}{*}{ c-LDL } & Normal & 185 & 86,4 & 86 & 71,7 & 70 & 68,0 & 0,000 \\
\hline & Alto & 29 & 13,6 & 34 & 28,3 & 33 & 32,0 & \\
\hline
\end{tabular}

C-HDL: colesterol ligado a lipoproteínas de alta densidad; C-LDL: colesterol ligado a lipoproteínas de baja densidad; IMC: índice de masa corporal.

a Prueba de $\mathrm{Chi}^{2}$.

se encuentran los hábitos alimentarios, la inactividad física, el sobrepeso y el perfil lipídico alterado.

Por fortuna, el IMC ha mostrado ser un indicador apropiado para estimar factores de riesgo cardiovascular. En este estudio se halló una diferencia significativa en la concentración de c-LDL de acuerdo con el IMC de los jóvenes, independiente del sexo, indicador que marca un perfil de riesgo para aquellos con sobrepeso y obesidad en comparación con los de peso adecuado. En las niñas, los valores de TG aumentaron y los de c-HDL disminuyeron a medida que se alteró la clasificación del IMC, lo cual representa un riesgo adicional. En general, la población estudiada de niños

Tabla 4 Frecuencia y cantidad promedio de frutas y verduras ingeridas por los jóvenes de 10 a 19 años de la empresa promotora de salud SURA. Medellín, Colombia

\begin{tabular}{|c|c|c|c|c|c|}
\hline \multicolumn{6}{|c|}{ Frecuencia y promedio de consumo de verduras y frutas } \\
\hline Verduras & $\%$ & Cantidad (g) & Frutas & $\%$ & Cantidad (g) \\
\hline Tomate & 32,0 & 44 & Mango & 14,1 & 105 \\
\hline Zanahoria & 31,9 & 21 & Limón & 13,7 & 28 \\
\hline Cebolla cabezona & 23,2 & 18 & Tomate de árbol & 11,6 & 46 \\
\hline Lechuga & 13,9 & 16 & Mora & 10,4 & 41 \\
\hline Cebolla común & 9,1 & 4 & Guayaba & 8,2 & 59 \\
\hline Repollo & 7,8 & 23 & Manzana & 7,8 & 125 \\
\hline Arveja & 5,6 & 29 & Banano & 7,6 & 82 \\
\hline Habichuela & 4,5 & 22 & Maracuyá & 5,6 & 116 \\
\hline Pimentón & 3,1 & 2 & Lulo & 4,8 & 55 \\
\hline Pepino & 2,3 & 50 & Naranja & 4,4 & 288 \\
\hline Auyama & 2,2 & 35 & Piña & 3,8 & 58 \\
\hline Champiñón & 1,6 & 26 & Fresa & 3,7 & 55 \\
\hline Remolacha & 1,4 & 44 & Papaya & 3,0 & 76 \\
\hline Apio & 1,0 & 17 & Mandarina & 3,0 & 183 \\
\hline Espinaca & 0,8 & 15 & Pera & 2,0 & 156 \\
\hline Brócoli & 0,6 & 46 & Granadilla & 2,0 & 41 \\
\hline Col & 0,6 & 31 & Uva & 1,8 & 59 \\
\hline Raíz china & 0,6 & 27 & Guanábana & 1,0 & 38 \\
\hline Calabacín & 0,4 & 93 & Zapote & 0,8 & 93 \\
\hline Coliflor & 0,4 & 30 & Sandía & 0,7 & 98 \\
\hline Cebolla puerro & 0,2 & 13 & Melón & 0,5 & 33 \\
\hline Brocoflor & 0,2 & 95 & Curuba & 0,4 & 25 \\
\hline Cidra & 0,1 & 10 & Durazno & 0,4 & 113 \\
\hline Calabaza & 0,1 & 45 & Uchuva & 0,4 & 30 \\
\hline
\end{tabular}


Tabla 5 Concentración de lípidos séricos de acuerdo con el consumo de frutas y verduras en jóvenes de 10 a 19 años de la empresa promotora de salud SURA. Medellín, Colombia

\begin{tabular}{|c|c|c|c|c|c|c|c|c|c|c|c|c|c|c|c|c|c|}
\hline \multirow[t]{2}{*}{ IMC } & \multirow{2}{*}{$\begin{array}{l}\text { Tertiles de } \\
\text { consumo }\end{array}$} & \multicolumn{4}{|c|}{ Valor del colesterol sérico } & \multicolumn{4}{|c|}{ Valor de los triglicéridos séricos } & \multicolumn{4}{|c|}{ Valor de las HDL séricas } & \multicolumn{4}{|c|}{ Valor de las LDL séricas } \\
\hline & & $\mathrm{n}$ & Media $\pm \mathrm{DE}$ & M & $\mathrm{p}^{\mathrm{a}}$ & $\mathrm{n}$ & Media $\pm \mathrm{DE}$ & M & $\mathrm{p}^{\mathrm{a}}$ & $\mathrm{n}$ & Media $\pm \mathrm{DE}$ & M & $\mathrm{p}^{\mathrm{a}}$ & $\mathrm{n}$ & Media $\pm \mathrm{DE}$ & M & $\mathrm{p}^{\mathrm{a}}$ \\
\hline \multirow[t]{7}{*}{ Obesidad } & Frutas & & & & & & & & & & & & & & & & \\
\hline & $\leq 1,62$ & 38 & $182,4 \pm 43,6$ & 174 & 0,859 & 38 & $110,9 \pm 53,7$ & 98 & 0,962 & 38 & $54,3 \pm 11,7$ & 53 & 0,152 & 38 & $91,5 \pm 30,7$ & 86 & 0,372 \\
\hline & 1,63 a 121,0 & 34 & $176,4 \pm 38,6$ & 180 & & 34 & $112,2 \pm 59,9$ & 103 & & 34 & $50 \pm 13,8$ & 48 & & 34 & $100,1 \pm 28,2$ & 99 & \\
\hline & $\begin{array}{l}>121,0 \\
\text { Verduras }\end{array}$ & 31 & $179,9 \pm 48,1$ & 165 & & 31 & $125,2 \pm 82,2$ & 100 & & 31 & $50,3 \pm 15,3$ & 48 & & 31 & $94 \pm 37$ & 86 & \\
\hline & $\leq 0,001$ & 43 & $187,5 \pm 43,9$ & 182 & 0,104 & 43 & $119,1 \pm 64,8$ & 100 & 0,268 & 43 & $52,4 \pm 14$ & 48 & 0,944 & 43 & $93,3 \pm 28,8$ & 93 & 0,443 \\
\hline & 0,1 a 42,08 & 26 & $181,5 \pm 38,1$ & 182 & & 26 & $103,3 \pm 66,7$ & 85 & & 26 & $51,7 \pm 14$ & 49 & & 26 & $91,4 \pm 37,4$ & 80 & \\
\hline & 42,09 a 91,2 & 34 & $168,4 \pm 44,4$ & 165 & & 34 & $120,6 \pm 64,9$ & 104 & & 34 & $50,7 \pm 13$ & 51 & & 34 & $100,2 \pm 31,4$ & 93 & \\
\hline \multirow[t]{7}{*}{ Sobrepeso } & Frutas & & & & & & & & & & & & & & & & \\
\hline & $\leq 1,62$ & 38 & $179,9 \pm 40,8$ & 172 & 0,993 & 38 & $118,6 \pm 78,7$ & 104 & $0,035^{b}$ & 38 & $53,5 \pm 11,2$ & 52 & 0,878 & 38 & $100,6 \pm 35,1$ & 96 & 0,212 \\
\hline & 1,63 a 121,0 & 38 & $180,1 \pm 42,9$ & 173 & & 38 & $123,4 \pm 59,7$ & 107 & & 38 & $52,1 \pm 13,1$ & 51 & & 38 & $88,8 \pm 32,6$ & 83 & \\
\hline & $\begin{array}{l}>121,0 \\
\text { Verduras }\end{array}$ & 44 & $182,2 \pm 51,1$ & 176 & & 44 & $96,6 \pm 65,3$ & 80 & & 44 & $53,8 \pm 13,1$ & 52 & & 44 & $98,3 \pm 35,4$ & 95 & \\
\hline & $\leq 0,001$ & 46 & $180,5 \pm 47,2$ & 176 & 0,957 & 46 & $112,6 \pm 76,1$ & 90 & 0,807 & 46 & $51,5 \pm 11,6$ & 51 & 0,260 & 46 & $100,2 \pm 42$ & 95 & 0,781 \\
\hline & 0,1 a 42,08 & 40 & $182,4 \pm 47$ & 173 & & 40 & $112,6 \pm 60$ & 108 & & 40 & $55 \pm 11,4$ & 52 & & 40 & $93,5 \pm 31,7$ & 89 & \\
\hline & 42,09 a 91,2 & 34 & $179,4 \pm 40,9$ & 172 & & 34 & $110,6 \pm 69,7$ & 94 & & 34 & $53,3 \pm 14,6$ & 49 & & 34 & $93,4 \pm 25,8$ & 97 & \\
\hline \multirow[t]{7}{*}{ Adecuado } & Frutas & & & & & & & & & & & & & & & & \\
\hline & $\leq 1,62$ & 68 & $171,3 \pm 40,7$ & 166 & 0,236 & 68 & $84,5 \pm 47,7$ & 72 & 0,721 & 68 & $55 \pm 12,6$ & 55 & 0,755 & 68 & $78,7 \pm 21,7$ & 75 & 0,186 \\
\hline & $\overline{1,63}$ a 121,0 & 74 & $179,9 \pm 37,2$ & 178 & & 74 & $85,8 \pm 38$ & 80 & & 74 & $55,4 \pm 13,7$ & 54 & & 74 & $84,2 \pm 26,5$ & 81 & \\
\hline & $\begin{array}{l}>121,0 \\
\text { Verduras }\end{array}$ & 72 & $172,4 \pm 41,2$ & 169 & & 72 & $92,5 \pm 67,3$ & 78 & & 72 & $56,6 \pm 14,4$ & 55 & & 72 & $76,5 \pm 22,2$ & 72 & \\
\hline & $\leq 0,001$ & 79 & $176,1 \pm 43,7$ & 173 & 0,126 & 79 & $92,8 \pm 48,9$ & 79 & 0,232 & 79 & $55,8 \pm 14,7$ & 55 & 0,232 & 79 & $78,1 \pm 22,7$ & 76 & 0,211 \\
\hline & $\overline{0}, 1$ a 42,08 & 55 & $181,3 \pm 38,9$ & 181 & & 55 & $80,1 \pm 30,6$ & 75 & & 55 & $57,8 \pm 12,8$ & 57 & & 55 & $85,1 \pm 24,5$ & 83 & \\
\hline & 42,09 a 91,2 & 80 & $168,7 \pm 35,4$ & 162 & & 80 & $87,8 \pm 65,6$ & 76 & & 80 & $54,1 \pm 12,8$ & 54 & & 80 & $78,1 \pm 24$ & 73 & \\
\hline
\end{tabular}

DE: desviación estándar; HDL: lipoproteínas de alta densidad; IMC: índice de masa corporal; LDL: lipoproteínas de baja densidad; M: mediana.

a Prueba de Kruskal-Wallis.

b Prueba U de Mann-Whitney. 
y niñas presentó un perfil lipídico de riesgo, considerando que la mitad de ellos tuvo hipercolesterolemia, uno de cada 3 , hipertrigliceridemia, uno de cada 5, c-LDL alto, y uno de cada 10, c-HDL bajo.

Estos resultados superan los reportes de otros países; por ejemplo, en un estudio realizado en adolescentes argentinos, se encontró que la prevalencia de hipercolesterolemia fue del 26,5\%, la de hipertrigliceridemia, del 20,1\%, y la de c-HDL bajo, del 17\%, es decir, los resultados en las 3 variables mencionadas fueron muy superiores ${ }^{10,20,21}$. Por otra parte, varios estudios realizados en escolares de diferentes países han mostrado resultados similares al nuestro en cuanto a la asociación del IMC con la concentración de lípidos sanguíneos, de tal manera que con el aumento del IMC se presentan mayores alteraciones en el perfil lipídico. En el estudio de Ella et al. ${ }^{12}$, se observó que los adolescentes obesos tuvieron casi el doble de riesgo de dislipidemia en comparación con los no obesos, y la incidencia de esta alteración entre obesos se podría reducir aproximadamente un $50 \%$ si el IMC se establece en el rango normal. Los resultados de ese estudio también mostraron que la incidencia de dislipidemia se puede reducir entre un 17 y un $19 \%$ si la obesidad se elimina 22,23 .

El estudio de Costa et al. mostró cómo el porcentaje de IMC en niños y adolescentes, distribuido por cuartiles, se asoció con diferentes factores de riesgo cardiovascular, entre ellos, la concentración de TG, cuyo promedio se incrementó a medida que aquel lo hacía, como también el c-HDL, cuya concentración disminuyó con el aumento en el porcentaje del IMC ${ }^{24}$. En nuestro estudio se observaron resultados similares en las niñas, quienes incrementaron los valores de TG a medida que se alteró el IMC, y el valor de C-HDL disminuyó al comparar las niñas obesas con las que presentaron un IMC adecuado. El c-HDL se considera un factor de protección y demuestra una relación inversa con el riesgo de enfermedad cardiovascular; algunos autores evidencian que por cada descenso de un $1 \%$ en la concentración de c-HDL, aumenta en un 2 a un $3 \%$ el riesgo cardiovascular ${ }^{25}$. Resultados diferentes tuvieron Dai et al., quienes no hallaron asociación entre el IMC y el incremento del CT, el c-LDL y los TG, pero reportaron que con el incremento de la grasa corporal se observó un aumento positivo y estadísticamente significativo en el perfil lipídico $^{26}$. Así mismo, el estudio HELENA ${ }^{27}$, que determinó el perfil lipídico detallado de una población adolescente europea teniendo en cuenta edad, sexo, madurez biológica, IMC, masa grasa y porcentaje de grasa corporal, demostró cómo en ambos sexos las concentraciones séricas de c-LDL y de TG se asociaron de manera positiva con el IMC y de forma negativa con las de c-HDL, resultados que coinciden con los de nuestro estudio para las niñas y niños en cuanto al c-LDL.

Aunque se reconoce la existencia de factores genéticos que predisponen a la obesidad, en la actualidad se destaca la alimentación como elemento determinante en la expresión génica; a su vez, el consumo de alimentos por parte de los adolescentes está influido por los padres, los amigos y la televisión. Algunos estudios han encontrado una asociación positiva entre la obesidad y el consumo de alimentos de alta densidad energética, y una asociación negativa entre el peso corporal y el consumo de frutas y verduras ${ }^{28}$.

Respecto a la ingesta de alimentos, el trabajo publicado en este número de la revista encontró una diferencia significativa en la concentración de TG de los niños con sobrepeso, en quienes el menor valor se observó en aquellos que se ubicaron en el tertil superior de consumo de frutas; no obstante, la mayoría de los jóvenes no reportó haber ingerido frutas y verduras el día anterior, y quienes consumieron estos alimentos tuvieron muy poca variedad y lo hicieron en cantidades muy por debajo de la recomendación de 400 a $500 \mathrm{~g}$ de frutas y verduras/día, que ha establecido la Organización Mundial de la Salud ${ }^{7}$. Estos resultados coinciden con los hallazgos de la Encuesta Nacional de la Situación Nutricional 2010, cuyo método de evaluación fue la frecuencia simple de alimentos y que encontraron que alrededor del $28 \%$ de los jóvenes ingirió diariamente frutas enteras, y alrededor de $14 \%$, verduras ${ }^{9}$; también concuerdan con los encontrados en Estados Unidos, donde se halló que aproximadamente la mitad de los adolescentes no consumen la cantidad de frutas y de verduras que se requieren como parte de una dieta saludable que contribuya a disminuir los riesgos en salud asociados con su bajo consumo ${ }^{28}$.

Contrario a los resultados de Lock et al. ${ }^{7}$ y Johnson-Taylor y Everhart $^{29}$, en niñas de 9 a 14 años de edad no encontraron relación entre la ingesta de jugos de frutas y verduras solas o en combinación y cambios en el valor z del IMC, y en los niños, no hubo relación entre el consumo de frutas y verduras y el sobrepeso. Además, una revisión realizada por Ledoux et al. concluyó que los estudios publicados en inglés no han logrado demostrar un efecto en la prevención de la adiposidad medida por IMC en los niños debido al consumo de frutas y verduras, pero sí se ha demostrado su efecto positivo en los adultos, que además está asociado con una menor ingesta de energía total ${ }^{6}$. Pese a lo anterior, es preciso considerar que el estudio de Lock et al. contempló todos los continentes para establecer las asociaciones, lo cual da un gran peso a sus resultados; además, se debe tener en cuenta que los hábitos alimentarios que se adquieren desde la niñez cobran importancia para el consumo habitual en la etapa adulta, aun cuando sus efectos no se pueden demostrar a corto plazo.

El seguimiento y la evaluación del comportamiento de los factores de riesgo potencial para el establecimiento de enfermedades crónicas no transmisibles en la población es imperativo, ya que estos pueden mostrar una tendencia a la variación en el tiempo. Por ejemplo, el estudio de Agirbasli et al. señaló que en Turquía hubo un aumento en la prevalencia de obesidad y sobrepeso significativo en niños y jóvenes de 8 a 18 años cuando se compararon 2 estudios, uno en 1989 y otro en 2008; resaltan un incremento cercano a 5 veces, especialmente en los adolescentes de 15 a 17 años. Respecto a los lípidos, estos autores no observaron una correlación con la prevalencia aumentada de obesidad, excepto para el c$\mathrm{HDL}$, que disminuyó significativamente entre adolescentes masculinos ${ }^{30}$.

Los jóvenes de nuestro estudio presentaron 2 de los criterios diagnósticos que se tienen en cuenta para definir el síndrome metabólico: TG altos y c-HDL bajo; a su vez, tuvieron un consumo de frutas y verduras muy inferior al de la recomendación $(400 \mathrm{~g})$, lo cual no favorece un perfil lipídico saludable. Al respecto, Bradlee et al., en un estudio de seguimiento durante 10 años en niñas, demostraron que al final de la adolescencia los patrones alimentarios caracterizados por una dieta saludable, que incluyó una ingesta alta y variada de frutas y verduras, fueron importantes predictores modificables de los lípidos en la adolescencia ${ }^{31}$. 
Las acciones para prevenir las enfermedades crónicas no transmisibles deben comenzar temprano en la vida del ser humano, ya que los factores de riesgo se instalan en la niñez y en la adolescencia y pueden predecir el riesgo en el adulto. Entre otros factores, se reconoce que la dislipidemia en los niños y adolescentes es un riesgo causal aceptado para el desarrollo de la placa arterial temprana; en tal sentido, la asociación que se demuestra entre el exceso de peso y la alteración del perfil lipídico a edades tempranas enfatiza la importancia del fomento de estilos de vida saludables que prevengan el sobrepeso y la obesidad.

La epidemia de exceso de peso en los jóvenes se asocia con alteraciones del perfil lipídico y malos hábitos de alimentación, que incluyen un bajo consumo de frutas y verduras, con muy poca variedad, y a su vez se convierten en factores de riesgo potencial para la aparición de enfermedades crónicas no transmisibles, todas modificables con intervenciones en las que participen el Estado, las instituciones educativas y prestadoras de servicios de salud, la industria de alimentos, las instituciones académicas y la comunidad misma, para contribuir a la disminución de estos riesgos en el ámbito familiar y social.

\section{Conclusión}

El sobrepeso y la obesidad se asocian con un perfil lipídico aterogénico. Se evidenció una asociación positiva entre el mayor consumo de frutas y la menor concentración de TG entre los participantes con sobrepeso; sin embargo, ninguno de los jóvenes ingirió la cantidad recomendada de frutas y verduras. Se recomiendan acciones interdisciplinarias e intersectoriales que promuevan estilos de vida saludables a edad temprana, con énfasis en la ingesta de una alimentación saludable y la prevención del exceso de peso.

\section{Financiación}

Los recursos para realizar la investigación fueron aportados por Vidarium, Centro de Investigación, Nutrición, Salud y Bienestar del Grupo Nutresa, la Universidad de Antioquia desde la Vicerrectoría de Investigación, la empresa promotora de salud SURA y la IPS Dinámica.

\section{Conflicto de intereses}

Gloria María Agudelo O. es investigadora de Vidarium, Centro de Investigación en Nutrición, Salud y Bienestar del Grupo Nutresa. Los demás autores declaran no tener ningún conflicto de intereses.

\section{Bibliografía}

1. Tohill BC. Dietary intake of fruit and vegetables and management of body weight. Atlanta, USA: World Health Organization; 2005 [actualizado 2005; consultado 2012]. Disponible en: http://www.who.int/dietphysicalactivity/publications/f\%26v weight_management.pdf

2. López-Jaramillo P. Cardiometabolic disease in Latin America: The role of fetal programming in response to maternal malnutrition. Rev Esp Cardiol. 2009;62:670-6.
3. Pan American Health Organization/World Health Organization (PAHO/WHO). Health information and analysis project (HSD/HA). Health situation in the Americas. Basic indicators 2012. Washington, D. C., United States of America: Pan American Health Organization, World Health Organization; 2012 [actualizado 2012; consultado 2012]. Disponible en: http://ais.paho.org/chi/brochures/2012/BI_2012_ENG.pdf

4. Daniels S, Greer F. Lipid screening and cardiovascular health in childhood. Pediatrics. 2008;122:198-208.

5. Calañas-Continente A, Bellido D. Bases científicas de una alimentación saludable. Rev Med Univ Navarra. 2006;50:7-14.

6. Ledoux TA, Hingle MD, Baranowski T. Relationship of fruit and vegetable intake with adiposity: A systematic review. Obes Rev. 2010;12:e143-50.

7. Lock K, Pomerleau J, Causer L, Altmann D, McKee M. The global burden of disease attributable to low consumption of fruit and vegetables: Implications for the global strategy on diet. Bull World Health Organ. 2005;83:100-8.

8. Instituto Colombiano de Bienestar Familiar, Profamilia, Instituto Nacional de Salud, Universidad de Antioquia. Encuesta nacional de la situación nutricional en Colombia 2005. Bogotá D. C.: Panamericana Formas e Impresos S. A.; 2006.

9. Instituto Colombiano de Bienestar Familiar. Encuesta nacional de la situación nutricional. Colombia 2010. Bogotá, D. C.: Davinci Editores \& CIA. S. en C.; 2011.

10. Uscátegui R, Álvarez M, Laguado I, Soler W, Martínez L, Arias R, et al. Factores de riesgo cardiovascular en niños de 6 a 18 años de Medellín (Colombia). An Pediatr (Barc). 2003;58:411-7.

11. Álvarez L, Mancilla L, González L, Isaza U. Perfil alimentario y nutricional de Medellín 2010. Libro Digital. Medellín: Alcaldía de Medellín, Universidad de Antioquia; 2010.

12. Ella N, Shehab D, Ismail M. Prevalence of overweight and obesity, and status of chronic non-communicable diseases and some related risk factors among Egyptian adolescents. J Diabetes Endocrinol. 2011;2:41-52.

13. WHO Multicentre Growth Reference Study Group. WHO child growth standards: Length/height-for-age, weight-for-age, weight-for-length, weight-for-height and body mass indexfor-age: Methods and development. Geneva: World Health Organization; 2006.

14. Manjarrés L. Métodos para precisar la recolección de la ingesta en estudios poblacionales. Perspect Nutr Humana. 2007;9:155-63.

15. National Cholesterol Education Panel. Report of the Expert Panel on Blood Cholesterol Levels in Children and Adolescents. NIH Publication No. 91-2732. Bethesda: National Institutes of Health; 1991.

16. Argüeso Armesto R, Díaz Díaz JL, Díaz Peromingo JA, Rodríguez González A, Castro Mao M, Diz-Lois F. Lípidos, colesterol y lipoproteínas. Galicia Clin. 2011;72 Supl 1:S7-17.

17. Asociación Médica Mundial. Declaración de Helsinki. Washington, D. C.: AMM; 2002 [actualizado 2002; consultado 2013]. Disponible en: http://www.relacionesinternacionales.info/ ojs/index.php?journal=Relaciones_Internacionales\&page= article\&op=viewFile\&path\%5B\%5D=179\&path\%5B\%5D= 165

18. Manjarrés L, Correa J. Programa de evaluación de ingesta dietética. Escuela de Nutrición y Dietética. $3 .^{a}$ ed. Medellín: Escuela de Nutrición y Dietética, Universidad de Antioquia; 2006.

19. Organización Panamericana de la Salud/Organización Mundial de la Salud. Perfil de los sistemas de salud. Colombia. Washington, D. C.: OPS; 2010 [actualizado 2010; consultado 2012]. Disponible en: http://new.paho.org/hq/dmdocuments/2010/ Perfil_Sistema_Salud-Colombia_2009.pdf

20. Pedrozo W, Bonneau G, Castillo M, Juárez M, Cardozo J. Valores de referencia y prevalencia de las alteraciones de perfil lipídico en adolescentes. Arch Argent Pediatr. 2010;108:107-15. 
21. Suárez P, Prin C, Luciani L, Turco M, D’Arrigo M, Rosillo I. Prevalencia de factores de riesgo de enfermedad cardiovascular: obesidad y perfil lipídico. An Pediatr (Barc). 2008;68:257-63.

22. Hosseini-Esfahani F, Mousavi Nasl Khameneh A, Mirmiran P, Ghanbarian A, Azizi F. Trends in risk factors for cardiovascular disease among iranian adolescents: The Tehran Lipid and Glucose Study, 1999-2008. J Epidemiol. 2011;21:319-28.

23. Ribas S, Santana da Siva L. Dislipidemia en escolares del sistema de educación privada de la ciudad de Belém. Arq Bras Cardiol. 2009;9:429-34.

24. Costa G, Horta N, Freire Z, Souza G, Machado L, Correia L, et al. Índice de masa corporal presenta buena correlación con el perfil proaterosclerótico en niños y adolescentes. Arq Bras Cardiol. 2009;93:256-61.

25. Barter PJ, Rye K-A. High density lipoproteins and coronary heart disease. Atherosclerosis. 1996;121:1-12.

26. Dai S, Fulton J, Harrist R, Grunbaum J, Steffen L, Labarthe D. Blood lipids in children: Age-related patterns and association with body-fat indices: Project HeartBeat! Am J Prev Med. 2009;37 1 Suppl:S56-64.
27. Spinneker A, Egert S, González-Gross M, Breidenassel C, Albers $\mathrm{U}$, Stoffel-Wagner B, et al. Lipid, lipoprotein and apolipoprotein profiles in European adolescents and its associations with gender, biological maturity and body fat-The HELENA Study. Eur J Clin Nutr. 2012;66:727-35.

28. Sharma M, Branscum P. Novel and emerging approaches to combat adolescent obesity. Adolesc Health Med Ther. 2010;1:9-19.

29. Johnson-Taylor WL, Everhart JE. Modifiable environmental and behavioral determinants of overweight among children and adolescents: Report of a workshop. Obesity (Silver Spring). 2006;14:929-66.

30. Agirbasli M, Adabag S, Ciliv G. Secular trends of blood pressure, body mass index, lipids and fasting glucose among children and adolescents in Turkey. Clin Obes. 2011;1:161-7.

31. Bradlee ML, Singer MR, Daniels SR, Moore LL. Eating patterns and lipid levels in older adolescent girls. Nutr Metab Cardiovasc Dis. 2013;23:196-204. 